\title{
Incidental Detection of Left External Iliac Artery Fibromuscular Dysplasia on Multidetector Computed Tomography
}

\author{
Richard Thomas* and Ayaz Aghayev \\ Department of Radiology, Brigham and Women's Hospital, USA
}

Submission: March 07, 2017; Published: May 26, 2017

*Corresponding author: Richard Thomas, Cardiovascular Imaging Program, Department of Radiology, Brigham and Women's Hospital, 75 Francis Street, Boston MA 02115, USA, Fax: (857) 307-2011; Tel: (857) 307-2030; Email: richardythms@gmail.com

Keywords: Fibromuscular dysplasia; External iliac artery; Computed tomography

Abbreviations: FMD: Fibromuscular Dysplasia; CT : Computed Tomography

\section{Introduction}

Fibromuscular dysplasia (FMD) is a condition predominantly affecting the renal and cervicocranial arteries. Isolated involvement of the iliac arteries is a rare manifestation of this disease. We describe a case of incidentally detected left external iliac artery fibromuscular dysplasia in a 53 year old female.

\section{Case Report}

A 53 year old female was referred for computed tomography (CT) angiogram of the abdomen and pelvis to evaluate the deep inferior epigastric artery perforators prior to breast reconstruction surgery. (Figure 1A \& 1B). The CT angiogram revealed a beaded appearance of the left external iliac artery. There was no significant stenosis or aneurysm of the artery. The remaining major arteries of the abdomen showed normal appearance. A diagnosis of isolated left external iliac artery fibromuscular dysplasia was made. As the patient was asymptomatic, this incidental finding did not warrant any further management.

\section{Discussion}

Fibromuscular dysplasia is a degenerative disease that affects medium sized vessels. It is most commonly seen in middle aged individuals with a 4:1 female preponderance. Classically the imaging appearance has been described as the 'string of beads sign' [1]. This is believed to be due to alternating segments of stenosis with aneurysms. Pathologically fibromuscular dysplasia has been categorized in to three different subtypes. The intimal and adventitial subtypes are seen on imaging as segments of diffuse stenosis or as segments

of aneurysmal dilatation. The medial subtype can be seen in the classic beaded appearance [2].

While renal arterial hypertension is the most common manifestation of FMD, cervicocranial involvement is the next most common presentation. Isolated iliac artery involvement is a rare manifestation. Such patients are usually detected incidentally to have the disease, during imaging done for other indications, as in our case. Iliac artery FMD may also present with features of vascular insufficiency. In this subset of patients endovascular treatment can relieve symptoms [3].

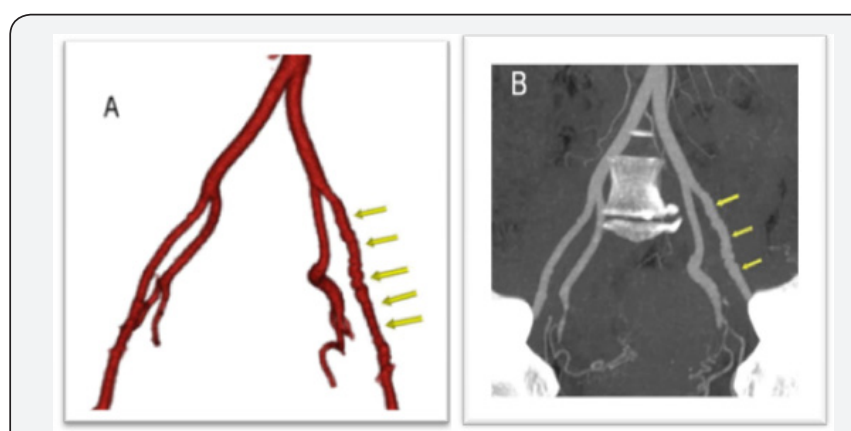

Figure 1: Coronal 3D post processed image $(A)$ and coronal maximum intensity projection image (B) show a beaded appearance of the left external iliac artery. No significant stenosis or aneurysm is seen.

With the advancements in imaging techniques, the diagnosis of fibromuscular dysplasia can be established with cross sectional imaging and most patients do not need 
histopathological confirmation. With its easy availability, high spatial resolution and ability to scan large anatomical regions rapidly, multidetector computed tomography is an excellent tool for the diagnosis of FMD. The ability to post process data and generate high quality 3D images is an added advantage of this modality [4]. For these reasons screening of the chest, abdomen and pelvis with CT angiography may be of benefit in patients with known FMD to identify other areas of involvement [5].

\section{References}

1. Plouin PF, Perdu J, La Batide-Alanore A, Boutouyrie P, GimenezRoqueplo AP, et al. (2007) Fibromuscular Dysplasia. Orphanet J Rare
Dis 2: 28 .

2. McCormack LJ, Poutasse EF, Meaney TF, Noto TJ, Dustan HP (1996) A pathologic-arteriographic correlation of renal arterial disease. Am Heart J 72(2): 188-198.

3. Ketha SS, Bjarnason H, Oderich GS, Misra S (2014) Clinical features and endovascular management of Iliac artery fibromuscular dysplasia. J Vasc Interv Radiol 25(6): 949-953.

4. Lewis S, Kadian-Dodov D, Bansal A, Lookstein RA (2016) Multimodality imaging of fibromuscular dysplasia. Abdom Radiol 41(10): 2048-2060.

5. Bolen MA, Brinza E, Renapurkar RD, Kim ESH, Gornik HL (2017) Screening CT angiography of the Aorta, visceral branch vessels and pelvic arteries in fibromuscular dysplasia. JACC Cardiovasc Imaging 10(5): 554-561.

\section{Your next submission with Juniper Publishers} will reach you the below assets 